\title{
Efecto de las variables de la gestión de diseño en el producto terminado
}

\section{Goiran, Andres Roque}

PhD candidate at Universitat Politècnica de València, Spain. angoiran@hotmail.com.

\section{Resumen}

La incorporación de métodos de siembra directa en lugar del laboreo tradicional, el aumento del valor de los granos, la demanda creciente de proteínas en el mundo, y los cambios de políticas económicas empujaron al sector agrícola argentino a una fuerte expansión productiva. La consecuencia directa de esta transformación fue que durante los últimos años aumentó la superficie sembrada y los rendimientos en la producción agrícola. Esto impulsó la demanda de máquinas agrícolas, y en consecuencia renacieron innumerables empresas fabricantes, surgiendo la necesidad de nuevos diseños que optimizaran el trabajo de mecanización con una única meta: bajar costos y tiempos.

Aplicando los conceptos de la Teoría General de Sistemas, en el proceso de diseño que se desarrolla en una pyme (pequeña y mediana empresa) agroindustrial interactúan distintas variables cuyo origen puede ser interno o externo. Esto influye en el éxito comercial de la organización, fundamento suficiente para analizarlo y comprenderlo.

En una primera instancia, en el presente trabajo se analizó cuáles son las variables características del proceso mencionado de la pequeña y mediana empresa agroindustrial de la Región Centro de la República Argentina. Como segunda instancia, se evaluó el impacto de las variables en el producto terminado, relacinándolas a través de un análisis retrospectivo con las no conformidades que surgieron en el periodo de garantía; de un grupo de máquinas elegidas para tal fin. Se comprobó que las influyen directamente pero en distintos grados en las no conformidades y en la percepción de confiabilidad del cliente. Se demostró que la comunicación y la gestión profesionalizada son las variable más influyentes. Se originaron como consecuencia dos realidades: por un lado la necesidad de una evolución tecnológica incorporando nuevas tecnologías en la gestión de la información y por otro lado, la necesidad de profesionalizar el proceso, demandando nuevos programas de capacitación adaptados a la realidad del rubro.

Palabras claves: confiabilidad, caracterización, productos agroindustriales, usuario, gestión de diseño. 


\begin{abstract}
The incorporation of direct sowing methods instead of traditional tillage, increasing the value of grains, the growing demand for protein in the world, and changes in economic policies pushed the agricultural sector to strong production growth, the direct consequence was that over recent years in the Argentine agricultural sector increased plantings and yields in agricultural production, this pushed the demand for agricultural machinery, reborn so many manufacturers dragging the need for new designs that will optimize the work of agricultural mechanization, with a single goal: cut costs and times.

Applying the concepts of General Systems Theory we think that in the design process that develops in an agroindustrial SME different variables interact whose origin can be internal or external, this undoubtedly influences the commercial success of the organization, foundation enough to analyze and understand. As a distinctive feature of it we can say that its identity depends on the type of organization, the product produced, the technological level, the market and history and idiosyncrasies of the company and its environment.

In this work in the first instance it will be elucidated what are the variables of the process mentioned an agribusiness small and medium industry of the Central Region of Argentina also their impact on the finished product so they were related through a retrospective analysis with the non-conformities that emerged in the warranty period of a group chosen for this purpose machines, proving that these directly influence but to varying degrees nonconformity and perceived reliability of the customer, being able to demonstrate that communication and professional management are the most influential variables, originating as a result two realities: on the one hand the need for technological evolution incorporating new technologies in information management such as integrated systems and on the other the need to professionalize the process, claiming this new training programs adapted to the reality in the field.
\end{abstract}

Keywords: Function, Variables, non conformities, Relationship

\title{
1. Introducción
}

El sistema de producción agrícola en la República Argentina ha sufrido profundas transformaciones en los últimos decenios. La incorporación de métodos de siembra directa en lugar del laboreo tradicional, el aumento del valor de los granos, la demanda creciente de proteínas en el mundo, y los cambios de políticas económicas empujaron al sector agrícola a una fuerte expansión productiva (Satorre, E. (2005). La consecuencia directa de esta transformación fue el aumento de la superficie sembrada y los rendimientos en la producción agrícola. Esto impulsó la demanda de máquinas agrícolas, y en consecuencia renacieron innumerables empresas fabricantes, surgiendo la necesidad de nuevos diseños que optimizaran el trabajo de mecanización con una única meta: bajar costos y tiempos.

Las máquinas que trabajan en la agricultura y sus partes componentes están predestinadas a cumplir las funciones asignadas en determinadas condiciones de producción y explotación técnica. El estado técnico de las maquinas durante el proceso de explotación cambia, así como cambian los valores de los parámetros desde lo nominal al límite (Shkiliova, et al., 2011). 
Una forma de caracterizar la maquinaria agrícola es aplicando el concepto de sistema. De acuerdo a la cantidad de sistemas que la conforman y el grado de nivel tecnológico de los mismos se definen: productos de alta tecnología (ej. tractores, cosechadoras), productos de media tecnología (ej. sembradoras, embolsadora de granos, extractora de granos), y productos de baja tecnología (ej. Implementos), existiendo una estrecha relación entre la confiabilidad y la caracterización tecnológica. Productos de alta tecnología son más confiables que productos media y baja por múltiples razones. Entre ellas, la gestión del diseño, procesos de fabricación, acceso a tecnología del conocimiento, nivel de facturación de la empresa de origen, organización empresarial, valor del producto, competencia, entre otros.

Según la Teoría General de Sistemas (Bertoglio, 1982), en una organización industrial existen variables de entrada que se transforman en un producto tangible por lo cual deben existir procesos que interactúan internamente y externamente. Uno de éstos procesos es el de diseño (Léon, et al., 2010), que influiría en el éxito comercial de la organización, fundamento suficiente para analizarlo y comprenderlo.

Como característica distintiva del proceso de diseño (pdd) su identidad depende del tipo de organización, del producto, del nivel tecnológico, del mercado y de la historia e idiosincrasia de la empresa y de su entorno.

En el presente trabajo, en una primera instancia se analizó cuales son las variables características de un proceso de diseño de la pequeña y mediana empresa (pyme) agroindustrial (productora de máquinas agrícolas de media y baja tecnología) de la Región Centro de la República Argentina. Como segunda instancia, se evaluó el impacto de las variables en el producto terminado, relacinándolas a través de un análisis retrospectivo con las no conformidades que surgieron en el período de garantía; de un grupo de máquinas elegidas para tal fin.

\section{Objetivos e Hipótesis}

El objetivo del trabajo fue detectar las variables propias del proceso de diseño característico de una pyme agroindustrial de la Región Centro de la República Argentina y analizar la influencia de las mismas en las no conformidades que surgen en el producto terminado en su período de garantía.

Se plantea como hipótesis que las variables intervinientes en el proceso de diseño influyen directamente en la percepción de confiabilidad del producto desde el punto de vista del cliente.

\section{Descripción del área de estudio.}

La Región Centro de la República Argentina, conformada por las provincias de Córdoba, Santa Fé y Entre Rios es una de las regiones más productivas del país con el 59\% de la producción nacional de granos de soja y el $95 \%$ de la producción nacional de aceites y harinas derivados de este cultivo. Esta región concentra el $17 \%$ del Producto Nacional Bruto y tiene una participación del 37\% en las exportaciones anuales ( INDEC censo 2010).

La organización empresarial de las mayoría de las empresas agroindustriales de la Región Centro tiene una base familiar. Éstas se concentran en nucleos territoriales. influenciadas directamente por el peso de la agricultura y de la ganadería, tratándose de un mercado complejo y heterogéneo según la particularidad de la zona. Este conjunto de empresas tiene un denominador común: una extensa localización territorial acorde con las demandas productivas regionales. La gran mayoría de las empresas son básicamente pymes, con un promedio de 30 años de antigüedad, cuyo tamaño va desde 10 hasta 300 empleados. El resto del mercado se distribuye en los segmentos de mayor valor económico y complejidad tecnológica. 


\subsection{Distribución porcentual de máquinas de media y baja tecnología fabricadas en la Región Centro}

Como se observa en la (fig.1) las fabricas de baja tecnología ( desmalesadoras, rastras, hileradoras, ) suman un 27\%, las de media tecnología ( acoplados tolvas, cultivadores, embolsadoras de granos, ensilladoras para granos y forrajes) un 51\% y de alta tecnología ( sembradoras, pulverizadoras, ) un $31 \%$.

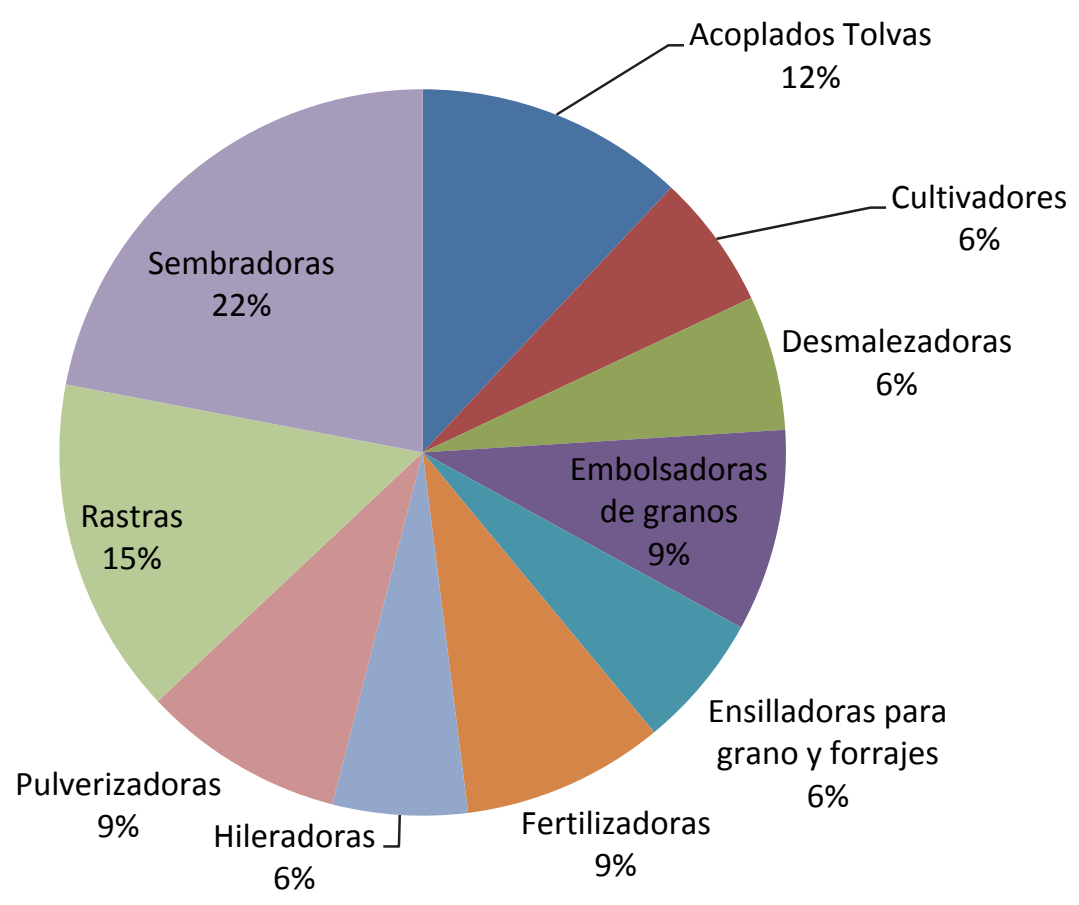

Fig. 1 Distribución porcentual de máquinas de media y baja tecnología fabricadas en la Región Centro.(fuente propia)

\section{Metodología}

\subsection{Determinación de variables características de una pyme agroindustrial}

Se realizaron encuestas con el método de entrevistas en profundidad a 10 expertos cuyo desempeño profesional está relacionado con el ámbito del proceso de diseño. La entrevista fue estandarizada abierta con un cuestionario de 33 preguntas. Las preguntas se elaboraron según los referenciales correspondientes de la norma VDA 6.3 (Aguilar, et al., 2006) sobre el método de auditoria de proceso de diseño. Se consideraron los siguientes ejes principales: entradas del diseño, planificación, herramientas del diseño, información, sistema de gestión de la calidad, resultado del proceso. La selección de los expertos fue multidisciplinar en cuanto a las funciones que desempeñan.

\subsection{Relevamiento de las No Conformidades de un grupo de máquinas agrícolas.}

La no conformidades se relevaron a través de un estudio del caso realizado en la empresa Micrón Fresar SRL productora de máquinas agrícolas de marca AKRON situada en la Región Centro de la República Argentina (provincia de Córdoba). La organización de la empresa representa el estándar típico de una empresa agroindustrial, sus productos son de media y baja tecnología. Sus procesos están certificados según la norma ISO 9001 y la mayoría de sus productos certifican las normas IRAM 8076 relativas a la seguridad de la maquinaria agrícola. 


\subsubsection{Datos relevantes de la firma}

Cantidad de personas empleadas: 150 (70\% directos y 30\% el resto indirectos). Capacidad productiva de la empresa: 120 máquinas mensuales. Modelo organizacional de sus líneas productivas: fabricación por líneas continúas de producción.

\subsubsection{Máquinas analizadas}

Se eligió un conjunto total de 450 máquinas producidas por la firma durante el año 2012, el seguimiento de las mismas finalizó en el año 2015 de modo que en la totalidad de las máquinas se cumplió el periodo de garantía otorgado por la empresa: 2,5 años.

Los reclamos procedente de los clientes se agruparon en: "mal funcionamiento" (se consignaron todos aquellos problemas que ocasionaron un mal funcionamiento en la máquinas), "roturas imprevistas", "error o faltante de piezas" (máquina despachada al cliente con piezas o subconjuntos faltantes), y "otros" (categoría donde la causa del reclamo no deriva a una no conformidad a la empresa ).

Posteriormente, se realizó un análisis para determinar las causas potenciales de las no conformidades, utilizando como metodología el Análisis de Modos Potenciales de Fallas (AMFE) (Stamatis,2003) ordenando las mismas según su índice potencial de riesgo ( IPR) según lo indica la metodología.

\subsection{Determinación de la influencia de las variables del proceso de diseño y las no conformidades.}

Se utilizó la metodología del análisis estructural (Cortezo, 2001) para evaluar la influencia de las variables y no conformidades. Ésta consistió en remitir al grupo de expertos una serie de cuestiones descriptivas del problema planteado. El proceso metodológico se repitió tres veces para asegurar la exactitud de las respuestas y disminuir los errores de interpretación. Los resultados se ordenaron en una matriz denominada estructural. Los valores discretos de 0 a 3 ponderaron la influencia de las variables entre sí, el 3 se le asigna a una influencia fuerte, 2 moderada, 1 leve y $0 \sin$ influencia.

El procesamiento de la matriz estructural se realizó con el software libre MICMAC (Posso, 2010), el cual permite determinar la influencia directa de un conjunto de variables respecto a una determinada.

\section{Resultados}

\subsection{Percepción de la confiabilidad del producto según el entorno}

La tabla 1 muestra el resultado de las encuestas realizada a los expertos, describiendo el eje temático, el promedio porcentual de concordancia de los mismos, las variables detectadas y su procedencia, es decir si ésta es externa, interna, salida y transversal a la organización.

Tabla 1: Variables intervinientes en la función del diseño

\begin{tabular}{|c|c|c|c|}
\hline Eje temático & Promedio & Variables & Procedencia \\
\hline & & Mercado & Externa \\
Entradas del diseño & \multirow{2}{*}{$86 \%$} & Clientes & Externa \\
& & Competencia & Externa \\
& & Exposiciones & Externa \\
\hline Planificación & $95 \%$ & Metodología & Interna \\
\hline
\end{tabular}


Efecto de las variables de la gestión de diseño en el producto terminado.

\begin{tabular}{|c|c|c|c|}
\hline & & $\begin{array}{l}\text { Verificación } \\
\text { Validación } \\
\text { Dirección }\end{array}$ & $\begin{array}{l}\text { Interna } \\
\text { Interna } \\
\text { Externa }\end{array}$ \\
\hline Herramientas de diseño & $80 \%$ & $\begin{array}{c}\text { Softwares de diseño } \\
\text { Métodos de cálculo } \\
\text { Adquisición de datos } \\
\text { Gestión profesionalizada } \\
\text { Diseño de Ensayos }\end{array}$ & $\begin{array}{l}\text { Interna } \\
\text { Interna } \\
\text { Interna } \\
\text { Interna } \\
\text { Interna }\end{array}$ \\
\hline Información & $96 \%$ & $\begin{array}{c}\text { Comunicación } \\
\text { Documentación Técnica } \\
\text { Relación Clientes }\end{array}$ & $\begin{array}{c}\text { Transversal } \\
\text { Interna } \\
\text { Externa }\end{array}$ \\
\hline $\begin{array}{l}\text { Sistema de Gestión de la } \\
\text { calidad }\end{array}$ & $75 \%$ & $\begin{array}{c}\text { Gestión No } \\
\text { conformidades } \\
\text { Política de calidad }\end{array}$ & $\begin{array}{l}\text { Transversal } \\
\text { Transversal }\end{array}$ \\
\hline Resultado del proceso & $98 \%$ & $\begin{array}{l}\text { Producto terminado } \\
\text { Confiabilidad }\end{array}$ & $\begin{array}{l}\text { Salida } \\
\text { Salida }\end{array}$ \\
\hline
\end{tabular}

\subsection{Variables sensibles de la percepción de la confiabilidad}

Los expertos consideraron a la variable confiabilidad como una variable de percepción. Si bien ésta se asocia en los primeros momentos de vida del producto con la trayectoria comercial y técnica de la organización, a medida que el producto se va consolidado en el mercado pasa a caracterizarlo. Según una encuesta realizada a clientes del rubro y región (ver artículo de Goirán en la presente edición), éstos construyen individualmente una percepción de confiabilidad a través de tres variables. Las cuales según el orden de importancia son: bajo índice de roturas imprevistas y desgastes prematuro (variables agrupadas por su origen mecánico), referencia de uso, disponibilidad en tiempo y forma del servicio técnico por parte de fabricante. (Fig. 2)

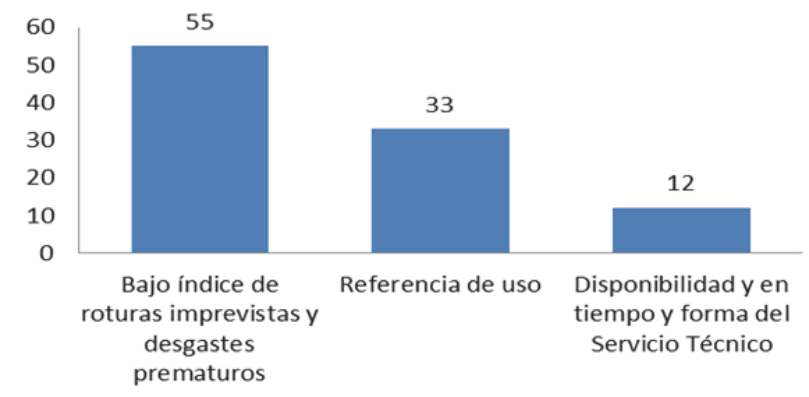

Fig. 2: Porcentaje de opiniones afirmativas respecto a las variables de confiabilidad del producto de acuerdo a la importancia de la prestación del servicio técnico. 


\subsection{No Conformidades del grupo de máquinas agrícolas}

\subsubsection{Cuantificación porcentual de las no conformidades}

Las no conformidades se conformaron de la siguiente forma: rotura imprevista $45 \%$, mal funcionamiento con el $30 \%$, Error o faltante de piezas el 25\%, y otros 2\% (Fig.3).

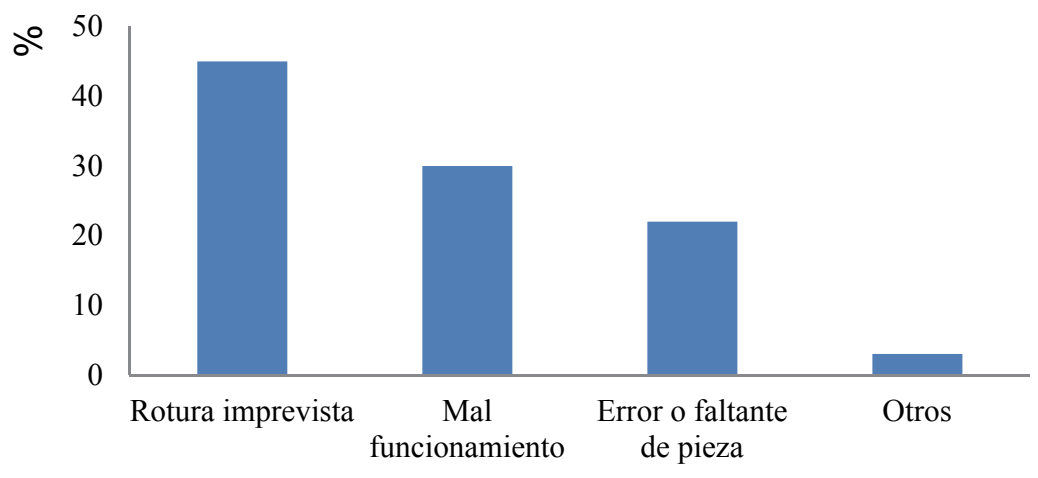

Fig.:3 Pareto de las no conformidades halladas.

\subsection{Análisis de las causas raíz de las no conformidades detectadas}

\subsubsection{No Conformidad: Mal Funcionamiento}

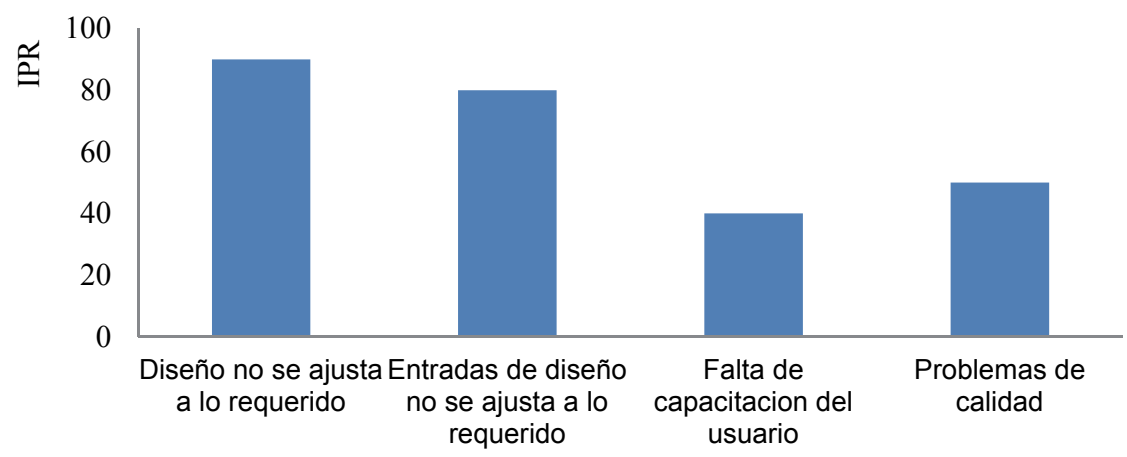

Fig.:4 Índice potencial de riesgo (IPR) de las causas raíz de la no conformidad mal funcionamiento.

El análisis de las causas raíz de la no conformidad Mal Funcionamiento (Fig. 4) da como resultado que la causa con mayor IPR( 90) fue el "diseño no se ajusta a lo requerido" seguido por la "entradas de diseño no se ajustan a lo requerido" con un IPR de 80 . Si bien a primera vista ambas parecen ser similares, la causa de mayor IPR se asocia a la gestión interna de la función de diseño y la siguiente con los requerimientos del mercado.

La causa "falta de capacitación del usuario" se puede atribuir a la falta de experticia en la operación de la máquina, reflejando esto último una deficiencia en la comunicación entre la empresa productora y el usuario. Por último se encuentra con un IPR de 40 "problemas de calidad y procesos", esta causa se 
relaciona directamente con el sistema de gestión de calidad y con la comunicación tecnológica entre la función diseño y la definición de los procesos productivos.

\subsubsection{No Conformidad: Rotura Imprevista}

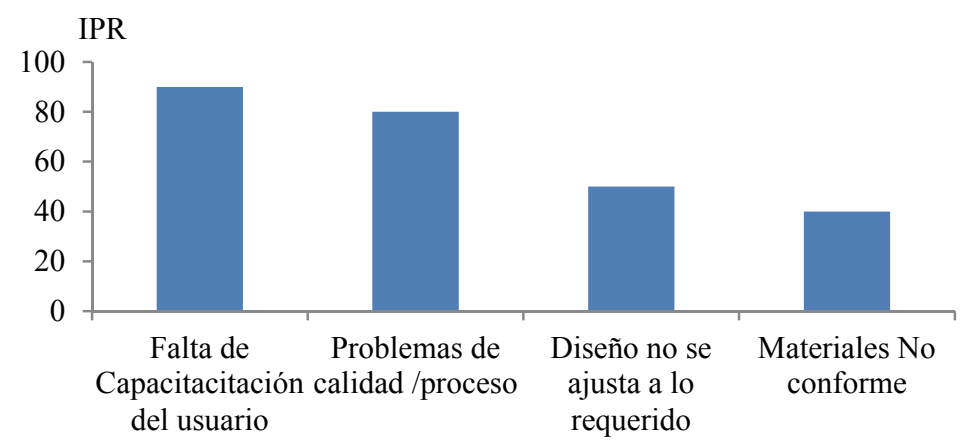

Fig.:5 Índice potencial de riesgo de las causas raiz de la no conformidad rotura imprevista.

El análisis de las causas raíz de la "rotura imprevista" (fig.5) da como resultado que, con un IPR de 90, la "falta de capacitación del usuario es la causa con mayor influencia, en segundo término "problemas de calidad y procesos" con un IPR de 80, en tercer lugar "diseño no se ajusta a lo requerido" con IPR de 50 y en un cuarto lugar materiales no conformes con IPR de 40.

Se destaca que la falta de capacitación del usuario radica en un problema de comunicación e información de la empresa sobre los modos de usos y capacidades funcionales de la máquina, en estos casos el usuario excede los límites de operatividad y la rompe.

\subsubsection{No Conformidad: Error faltante de Montaje}

El análisis de la no conformidad "error o faltante de pieza" (fig. 6) da como resultado que las primer causa raíz se debe a "instrucciones de proceso incorrectas" con un IPR de 120, en segundo lugar "falta de capacitación al operario" con un IPR de 80 y en tercer "planimetría incorrecta". Estas causas tienen denominador común relacionado con el manejo de la información y comunicación en la empresa. En cuarto lugar, "falta de control de calidad" con un IPR de 30, esta causa está relacionada con el sistema de gestión de calidad de la empresa. 


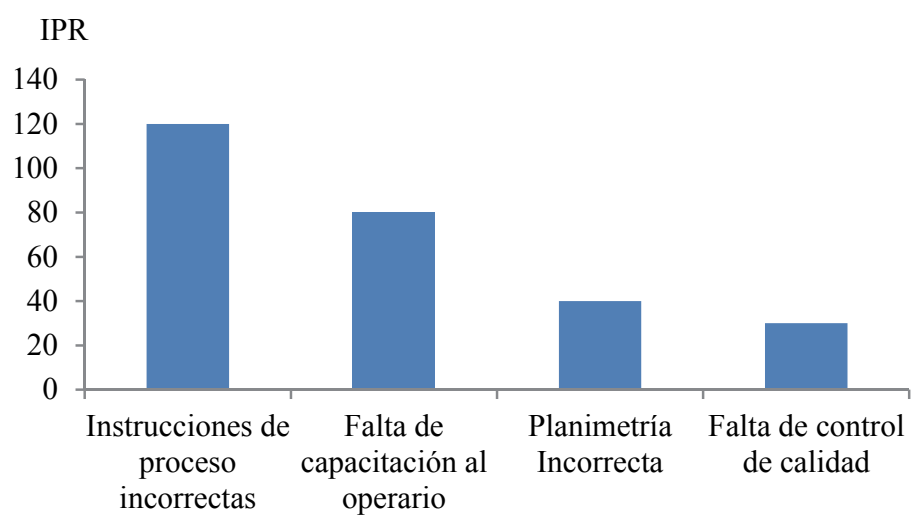

Fig. 6: Índice potencial de riesgo de las causas raiz de la no conformidad "Error faltante de Montaje"

\subsection{Análisis de la relación causa raíz y las variables de mayor influencia}

El resultado del análisis se expresa en la tabla 2, en ella se puede observar el nivel de influencia entre distintas variables del PDD y las causas raíces de las no conformidades.

En el gráfico de la figura 7 se muestra el nivel de influencia porcentual de las variables características del proceso de diseño calculado a través del software MICMAC .Se observa que las variable comunicación, gestión profesionalizada y documentación técnica tienen la mayor influencia con un el porcentual acumulado que no supera el $80 \%$. 
Efecto de las variables de la gestión de diseño en el producto terminado.

Tabla 2 Cuadro relación causa raíz variables de mayor influencia

\begin{tabular}{|c|c|c|c|c|c|}
\hline \multirow{2}{*}{ No conformidad } & \multirow{2}{*}{ Causa Raíz } & \multirow{2}{*}{ Variables } & \multicolumn{3}{|c|}{ Influencia } \\
\hline & & & 3 & 2 & 1 \\
\hline \multirow{12}{*}{ Mal Funcionamiento } & \multirow{4}{*}{$\begin{array}{l}\text { Diseño no se } \\
\text { ajusta a lo } \\
\text { requerido }\end{array}$} & Gestión profesionalizada & $\mathrm{X}$ & & \\
\hline & & Diseño de ensayos & & $\mathrm{X}$ & \\
\hline & & Relación clientes & $\mathrm{X}$ & & \\
\hline & & Comunicación & $\mathrm{X}$ & & \\
\hline & \multirow{6}{*}{$\begin{array}{c}\text { Entradas del } \\
\text { diseño no se } \\
\text { ajusta a lo } \\
\text { requerido } \\
\text { Falta de } \\
\text { capacitación del } \\
\text { usuario }\end{array}$} & Comunicación & $\mathrm{X}$ & & \\
\hline & & Dirección & & $\mathrm{X}$ & \\
\hline & & Comunicación & $\mathrm{X}$ & & \\
\hline & & Relación clientes & $\mathrm{X}$ & & \\
\hline & & Política de la calidad & & $\mathrm{X}$ & \\
\hline & & Dirección & $\mathrm{X}$ & & \\
\hline & \multirow{2}{*}{$\begin{array}{c}\text { Problemas de } \\
\text { calidad o } \\
\text { procesos }\end{array}$} & Comunicación & $\mathrm{X}$ & & \\
\hline & & Documentación Técnica & & $\mathrm{X}$ & \\
\hline \multirow{4}{*}{ Rotura imprevista } & \multirow{4}{*}{$\begin{array}{l}\text { Materiales no } \\
\text { conformes }\end{array}$} & Métodos de cálculo & & $\mathrm{X}$ & \\
\hline & & Adquisición de datos & & & $\mathrm{X}$ \\
\hline & & Gestión profesionalizada & $\mathrm{X}$ & & \\
\hline & & Diseño de Ensayos & & $\mathrm{X}$ & \\
\hline \multirow{8}{*}{$\begin{array}{l}\text { Error o faltante de } \\
\text { montaje }\end{array}$} & \multirow{3}{*}{$\begin{array}{c}\text { Instrucciones de } \\
\text { proceso } \\
\text { incorrectas. }\end{array}$} & Comunicación & $\mathrm{X}$ & & \\
\hline & & Documentación Técnica & $\mathrm{X}$ & & \\
\hline & & $\begin{array}{c}\text { Relación otras áreas de } \\
\text { la organización }\end{array}$ & $\mathrm{X}$ & & \\
\hline & \multirow{3}{*}{$\begin{array}{c}\text { Falta de } \\
\text { capacitación al } \\
\text { operario. }\end{array}$} & Comunicación & $\mathrm{X}$ & & \\
\hline & & Documentación Técnica & & $\mathrm{X}$ & \\
\hline & & $\begin{array}{l}\text { Relación otras áreas de } \\
\text { la organización }\end{array}$ & $\mathrm{X}$ & & \\
\hline & $\begin{array}{c}\text { Planimetría } \\
\text { incorrecta }\end{array}$ & Gestión profesionalizada & $\mathrm{X}$ & & \\
\hline & $\begin{array}{c}\text { Falta de control } \\
\text { de calidad }\end{array}$ & ----- & - & - & - \\
\hline
\end{tabular}

Fig. 7 Nivel de influencia porcentual de las distintas variables en las no conformidades detectada

\section{Discusión}

El proceso de diseño en una organización tiene relación directa con el producto terminado no solo en el aspecto técnico sino también económico. Al considerar que éste es un proceso dentro de la organización estará constituido por variables de distintos tipos que interactúan internamente y externamente con mayor o menor influencia pero todas sin lugar a dudas influyen en el resultado. no solamente en las no conformidades que se van produciendo a lo largo durante el período de garantía del producto sino que también influyen la percepción de confiabilidad del cliente factor clave y decisivo en la decisión de compra de una máquina. 
Comunicación y gestión profesionalizada se pueden considerar como variables claves en el PDD, ésta situación plantea un gran desafío en las organizaciónes que es la de en primer lugar sobrevivir en un mercado tan competitivo y por el otro lade crecer por lo que tendrán que ajustar los canales y formas de comunicación además de reveer los procesos de profesionalización.

La dirección de éstas pymes también influyen en el PDD, éstas en términos generales deciden que fabricar, como relacionarse con los clientes y cuales son las políticas de calidad a implementar, muchas veces por razones de mercado, la dirección debe prioriza el factor económico teniendo en esos caso una mayor influencia en los procesos internos del PDD.

\section{Conclusiones}

Las variables comunicación, gestión profesionalizada y documentación técnica son las variables de mayor influencia aunque no hay un predominio categórico respecto a las demás variables. Sería ilógico pensar que estamos antes la presencia de variables absolutamente independientes, en mayor o menor medida hay una influencia entre ellas, por ejemplo la comunicación tiene relación directa con la profesionalización de área, a su vez la documentación técnica es una forma de comunicación.

\section{Biblografía}

BERTOGLIO, O. \& JOHANSEN, O.(1982) Introducción a la teoría general de sistemas. Editorial Limusa. Mexico. ISBN 968-18-1567-X.

STAMATIS, D, (2003). Failure mode and effect analysis: FMEA from theory to execution. ASQ Quality Press.

CORTEZO, J. (2001). Introducción a la prospectiva: metodologias, fases y explotación de resultados. $<$ http://www.minetur.gob.es/Publicaciones/Publicacionesperiodicas/EconomiaIndustrial/RevistaEconomia Industrial/342/1JesusRodriguez.pdf $>$ [Consulta: 20 de Mayo de 2016]

LEÓN, A; RIVERA, D; NARIÑO, A. (2010). Relevancia de la Gestión por Procesos en la Planificación Estratégica y la Mejora Continua. $<$ http://www.altagestion.com.co/boletines/mailing2015/redes_sociales/doc/0702_DOCUMENTO_RelevanciaGPP_2 0150708.pdf> [Consulta: 20 de Mayo de 2016].

POSSO, D. \& MURIEL, S. (2010). Análisis estructural: un apoyo para el modelado con dinámica de sistemas. $<$ http://www.bdigital.unal.edu.co/28807/1/26663-93574-1-PB.pdf $>$ [Consulta: 20 de Mayo de 2016]

SATORRE, E. (2005). Cambios tecnológicos en la agricultura argentina actual. $<$ http://www.agrotecnicounne.com.ar/biblioteca/Anexo\%203-\%20Satorre\%20en\%20colores-1.pdf> [Consulta: 20 de Mayo de 2016].

SHKILIOVA, L. \& FERNANDEZ-SANCHEZ, M. (2011). Sistemas de Mantenimiento Técnico y Reparaciones y su aplicación en la Agricultura. $\quad<$ http://scielo.sld.cu/scielo.php?pid=S207100542011000100013\&script=sci_arttext $>$ [Consulta: 20 de Mayo de 2016].

AGUILAR MENDOZA, M. (2006). Auditoría de procesos VDA 6.3. Universidad Autónoma de Nuevo León. $<$ http://eprints.uanl.mx/1689/1/1080167883.pdf> [Consulta: 20 de Mayo de 2016].

INSTITUTO NACIONAL DE ESTADISTICAS Y CENSOS. Censo 2010. $<$ http://www.indec.mecon.ar/> Consulta: 20 de Mayo de 2016]. 